
\title{
25 Research Suare \\ Effect of octahedron tilt on structure and magnetic properties of bismuth ferrite
}

\author{
Yang Hong \\ Harbin Institute of Technology \\ Jun Li ( $\nabla$ lijuna@hit.edu.cn ) \\ Harbin Institute of Technology https://orcid.org/0000-0002-7645-1997 \\ Han Bai \\ Harbin Institute of Technology \\ Zhenjia Song \\ Harbin Institute of Technology \\ Ming Wang \\ Harbin Institute of Technology \\ Zhongxiang Zhou \\ Harbin Institute of Technology
}

\section{Rapid Communication}

Keywords: bismuth ferrite; magnetism; octahedron tilt.

Posted Date: April 29th, 2020

DOl: https://doi.org/10.21203/rs.3.rs-24754/v1

License: (c) (i) This work is licensed under a Creative Commons Attribution 4.0 International License. Read Full License

Version of Record: A version of this preprint was published at Journal of Advanced Ceramics on September 25th, 2020. See the published version at https://doi.org/10.1007/s40145-020-0398-1. 


\section{Abstract}

Multiferroic trivalent ions doped $\mathrm{BiFeO}_{3}$ ceramics were synthesized using the rapid liquid state method. The trivalent ions $\left(\mathrm{Sm}^{3+}, \mathrm{Gd}^{3+}, \mathrm{Y}^{3+}\right)$ doping causes structural distortion without changing the rhombohedral structure. Raman analysis shows that the effect of doping on $E$ modes is greater than $A_{1}$ modes, and $\mathrm{FeO}_{6}$ octahedron is regulated by ions doping. $\mathrm{M}-\mathrm{H}$ hysteresis loops show $\mathrm{A}$-site trivalent ions doped samples exhibit improved magnetism compared with pure $\mathrm{BiFeO}_{3}$ since the suppressed spiral spin structure. Doped samples exhibited natural resonance around $16 \sim 17 \mathrm{GHz}$ due to the changes in dielectric loss caused by the tilt of the $\mathrm{FeO}_{6}$ octahedron.

\section{Introduction}

Multiferroic materials are one of the most studied materials in recent years due to their unique properties, which exhibit more than two ferroic orders (such as ferroelectricity, ferromagnetism, ferroelastic etc.). More than two orders can be coupled under certain conditions, exhibiting remarkable physical properties and excellent application potential [1-3]. Multiferroic materials are not only helpful for the applications but also provide a platform for exploring interesting effects like magnetoelectric effect, piezoelectric effect, and so on.

Among various known multiferroic materials, bismuth ferrite $\left(\mathrm{BiFeO}_{3}\right)$ is a widely investigated multiferroic material, in which ferroelectricity $\left(T_{\mathrm{C}} \approx 1100 \mathrm{~K}\right)$ and ferromagnetism $\left(T_{\mathrm{N}} \approx 640 \mathrm{~K}\right)$ coexist at room temperature $[4,5]$. The crystallographic structure of $\mathrm{BiFeO}_{3}$ is rhombohedrally distorted perovskite structure with the space group $R 3 c$. The unit cell has a lattice parameter of $a=3.965 \AA$ and a rhombohedral angle of $\sim 89.3-89.48^{\circ}$, and the oxygen octahedron is distorted with minimum and maxmum 0-0 distances of 2.710 and $3.015 \AA$, respectively, and rotated by about $13.8^{\circ}$ around the [111] axis [6]. $\mathrm{BiFeO}_{3}$ is classified as a G-type antiferromagnet below $T_{\mathrm{N}}$ at the magnetic point of view, the combine action of exchange and spin-orbit interactions produce spin canting away from perfect antiferromagnetic ordering. The canted spin structure exhibits a space-modulated spiral structure (SMSS) with a period length of $62 \mathrm{~nm}$, thereby resulting in a helimagnetic structure and a vanishing magnetization in the bulk $[7,8]$.

The SMSS ordering in $\mathrm{BiFeO}_{3}$ is stable, and it persists when the temperature varies from $4 \mathrm{~K}$ to the Neel temperature [9]. The modification of the spin structure is the key issue for the realization of $\mathrm{BiFeO}_{3}$-based materials. Many attempts to add ferromagnetic properties to the $\mathrm{BiFeO}_{3}$ compounds by $\mathrm{A}$ or $\mathrm{B}$ site substitutions were made. Neutron diffraction studies on $\mathrm{Bi}_{1-x} \mathrm{La}_{x} \mathrm{FeO}_{3}$ show that the $\mathrm{SMSS}$ modulation period in $\mathrm{BiFeO}_{3}$ grows with $\mathrm{La}[10]$. The researchers have found that the leakage current of $\mathrm{BFO}$ can be reduced by substituting appropriate element. To improve the electrical and magnetic properties of the BFO, several research groups have attempted to modify with $3+$ valence ions of $\mathrm{Nd}^{3+}, \mathrm{Dy}^{3+}$ or $\mathrm{Pr}^{3+}$ at the 
A-site of $\mathrm{BFO}$ [11-13]. The spin-coated $\mathrm{Y}$-doped $\mathrm{BiFeO}_{3}$ film exhibits low field saturation magnetization by suppressing the spiral spin modulated periodicity due to $\mathrm{FeO}_{6}$ octahedral distortion [14].

In present work, we study structure, magnetic properties and microwave electromagnetic parameters of $\mathrm{BiFeO}_{3}$ based ceramics. A-site trivalent ions doping tilted the $\mathrm{FeO}_{6}$ octahedron in the lattice and changed the space-modulated spiral structure, leading to the improved ferromagnetism of ceramics. Doped samples exhibited enhanced permittivity accompanied natural resonance around $16 \sim 17 \mathrm{GHz}$, the detailed mechanism has been explained with dielectric loss and defects changed.

\section{Experiment}

Polycrystalline $\mathrm{BiFeO}_{3}$ and $\mathrm{Bi}_{0.95} M_{0.05} \mathrm{FeO}_{3}(M=\mathrm{Sm}, \mathrm{Gd}, \mathrm{Y}$ ) samples (abbreviated as $\mathrm{BFO}, \mathrm{BSFO}, \mathrm{BGFO}$ and $\mathrm{BYFO}$ ) were prepared by the rapid liquid state method. Stoichiometric ratios of $\mathrm{Bi}_{2} \mathrm{O}_{3}, \mathrm{Fe}_{2} \mathrm{O}_{3}, \mathrm{Sm}_{2} \mathrm{O}_{3}$, $\mathrm{Gd}_{2} \mathrm{O}_{3}, \mathrm{Y}_{2} \mathrm{O}_{3}$ were mixed with agate balls for $24 \mathrm{~h}$ using alcohol as the solvent. Each component has an increase of $2.5 \% \mathrm{Bi}_{2} \mathrm{O}_{3}$ on the base of the stoichiometric ratio, taking into account the volatilization characteristic of $\mathrm{Bi}_{2} \mathrm{O}_{3}$. The powders were pressed into pellets of $8 \mathrm{~mm}$ in diameter under $500 \mathrm{MPa}$ rapidly sintered in air at $800^{\circ} \mathrm{C}$ for $20 \mathrm{~min}$, and then took a rapid air quenching process.

The phase composition and crystalline structures of the samples were analyzed using X-ray diffraction (XRD, Panalytical X'PERT PRO MPD, with Cu Ka radiation). Morphologies were examined using field emission scanning electron microscopy (SEM, ZEISS Merlin Compact). Raman measurement was performed using laser confocal Raman spectrometer (inVia-Reflex, Renishaw). The magnetic properties were investigated by a vibrating sample magnetometer (VSM). The electromagnetic parameters were measured from 2 to $18 \mathrm{GHz}$ with a vector network analyzer (Keysight Technogies N5234A).

\section{Results And Discussion}

In order to evaluate the phase formation and crystallinity of the samples, X-ray diffraction study was performed, and the effect of the substitution of doped ions for $\mathrm{Bi}^{3+}$ ion on the structure of $\mathrm{BiFeO}_{3}$ was investigated by Rietveld refinement, which was performed by using the GSAS program. Figure 1 shows the $x$-ray diffraction (XRD) patterns and the refinement curves of the as-prepared samples. All the exhibited peaks of four samples were well indexed with $\mathrm{BiFeO}_{3}$ (ICDD-PDF No.71-2494) and have rhombohedral $R 3 c$ structure. The corresponding lattice parameters and fitting factors were listed in Table 1, ions introduction played a significant effect on the crystal structure. Since the radius of the doped ions is smaller than that of the bismuth ion (Six coordination ionic radius: $\mathrm{rBi}^{3+}=0.103 \mathrm{~nm}, \mathrm{rSm}^{3+}$ $=0.0958 \mathrm{~nm}, \mathrm{rGd}^{3+}=0.0938 \mathrm{~nm}, \mathrm{rY}^{3+}=0.0900 \mathrm{~nm}$ ), the unit cell volume shrinks after doping. In addition, affected by changes of ion radius in the sublattice, the state of the local electronic cloud changed, so the bond length $(\mathrm{Fe}-\mathrm{O})$ and the bond angle $(\mathrm{Fe}-\mathrm{O}-\mathrm{Fe})$ have changed in the $\mathrm{FeO}_{6}$ octahedron, and the $\mathrm{FeO}_{6}$ octahedron distorted. 
The angle of rotation of oxygen octahedron is a typical structural parameter of BFO. For cubic perovskite with standard matching ions, this rotation angle is zero. However, in BFO rhombohedral perovskite system, the rotation angle is nonzero for the influence of overlap between the electronic clouds of ions. 
Table 1

Refined lattice parameters and structure fitting factors.

\begin{tabular}{|lllll|}
\hline Parameters & BFO & BSFO & BGFO & BYFO \\
\hline$a(\AA)$ & 5.5795 & 5.5798 & 5.5788 & 5.5794 \\
\hline$c(\AA)$ & 13.8709 & 13.8623 & 13.8609 & 13.8687 \\
\hline$V\left(\AA^{3}\right)$ & 373.960 & 373.767 & 373.594 & 373.893 \\
\hline $\mathrm{Fe} z$ & 0.2222 & 0.2456 & 0.2707 & 0.2316 \\
\hline $\mathrm{O} x$ & 0.4520 & 0.4360 & 0.4440 & 0.4277 \\
\hline $\mathrm{O} y$ & 0.0227 & 0.0003 & 0.0092 & -0.0130 \\
\hline $\mathrm{O} z$ & 0.9554 & 0.9807 & 1.0020 & 0.9995 \\
\hline $\mathrm{Fe}-\mathrm{O}(1)$ bond length $(\AA)$ & 2.071 & 2.138 & 2.137 & 2.122 \\
\hline $\mathrm{Fe}-\mathrm{O}(2)$ bond length $(\AA)$ & 1.980 & 1.905 & 1.912 & 1.939 \\
\hline Fe-O-Fe bond angle $\left(^{\circ}\right)$ & 165.7 & 170.3 & 166.5 & 166.5 \\
\hline$R_{\mathrm{wp}}$ & $7.42 \%$ & $7.63 \%$ & $6.99 \%$ & $7.25 \%$ \\
\hline$R_{\mathrm{p}}$ & $5.80 \%$ & $5.87 \%$ & $5.51 \%$ & $5.47 \%$ \\
\hline$\chi^{2}$ & 2.086 & 2.997 & 2.480 & 2.788 \\
\hline
\end{tabular}

The angle of rotation of oxygen octahedron is a typical structural parameter of BFO. For cubic perovskite with standard matching ions, this rotation angle is zero. However, in BFO rhombohedral perovskite system, the rotation angle is nonzero for the influence of overlap between the electronic clouds of ions.

In the doped system of perovskite structure compounds, the tolerance factor, defined as $\tau=\frac{r_{A}+r_{O}}{\sqrt{2}\left(r_{B}+r_{O}\right)}$, is usually used to depict the stable extent of the structure. $r_{A}, r_{B}$, and $r_{O}$ stand for the ionic radius of A-site, $\mathrm{B}$-site, and O-site in the $\mathrm{ABO}_{3}$, respectively [15]. The tolerance factor $\tau$ is 0.8404 for the pure BFO, and it is $0.8391,0.8388,0.8381$ for BSFO, BGFO, and BYFO, respectively. It can be seen that the structure of the four components is relatively stable. The smaller the tolerance factor, the more severely is the bucking between the oxygen octahedron. The smaller A-site ions can not fill the empty space fully leading to the distorted octahedron, shrinking the unit cell space. The octahedral tilt is $\sim 11-14^{\circ}$ along the [111] triple axis and the related Fe-O-Fe band angle is $154-156^{\circ}$ [16].

Scanning electron microscopy technology was used in pure and doped BFO samples, as shown in Fig. 2. All the samples exhibit distinct morphologies and boundaries, and a certain amount of pores exist in the ceramics. In order to avoid heterogeneous phases during sintering, rapid liquid phase sintering and air quenching were adopted, this has caused a change in the porosity of the ceramics. The grain size of pure BFO was found to be $1-3 \mu \mathrm{m}$, with the introduction of doped ions, the average grain sizes of doped ceramics are smaller than that of BFO, which are around 0.5-1 $\mu \mathrm{m}$. The difference in ionic radii of A-site lead to shrink of unit cell which can hinder crystallite nucleation, and the grain sizes are smaller in the macro perspective. 
The crystal structure of BFO is a distorted rhombohedral perovskite at room temperature, which belongs to space group $R 3 C$. According to group theory, the multiferroic BFO exhibits 13 optical phonon modes, expressed by the equation as (see Equation in the Supplemental Files)

$$
\Gamma_{\text {Rhombohedral, R3c }}=4 A_{1}+9 E
$$

where, The $A_{1}$ modes polarized along and the doubly degenerate $E$ modes polarized in the x-y plane are both Raman and IR active. The peaks, which represent the Raman modes, are obtained by the Raman spectra and decomposing them into individual components, as shown in Fig. 3. In our polycrystalline ceramics, all the 13 Raman active modes were observed, which matched well with the above structure. $\mathrm{Bi}$ atoms participate in low-frequency modes below $A_{1}$ (TO1), whereas 0 atoms dominate in modes above $E(\mathrm{TO} 4)$. Fe atoms are involved mainly in modes between $E(\mathrm{TO} 2)$ and $E(\mathrm{TO} 4)$, but also contribute to the development of some higher-wavenumber modes.

We observed all Raman modes of the rhombohedral structure of $\mathrm{BiFeO}_{3}$ predicted by theory from the spectrum, and labeled them in Fig. 3. Table 2 exhibits the comparison of the Raman modes positions after being deconvoluted of different component samples. By comparing Raman shifts of different modes, it is found that the doping has a certain effect on the $E$ modes, but has little effect on the $A_{1}$ modes. 
Table 2

Comparison of the Raman modes positions of different component samples.

\begin{tabular}{|c|c|c|c|c|}
\hline Raman modes & BFO & BSFO & BGFO & BYFO \\
\hline$A_{1}(\mathrm{TO} 1)$ & 137 & 138 & 140 & 140 \\
\hline$A_{1}(\mathrm{TO} 2)$ & 170 & 171 & 172 & 173 \\
\hline$A_{1}(\mathrm{TO} 3)$ & 218 & 224 & 225 & 224 \\
\hline$A_{1}(\mathrm{TO} 4)$ & 453 & 450 & 450 & 456 \\
\hline$E(\mathrm{TO} 1)$ & 75 & 76 & 76 & 76 \\
\hline$E(\mathrm{TO} 2)$ & 121 & 118 & 123 & 121 \\
\hline$E(\mathrm{TO} 3)$ & 258 & 258 & 266 & 264 \\
\hline$E(\mathrm{TO} 4)$ & 284 & 279 & 295 & 288 \\
\hline$E(\mathrm{TO5})$ & 340 & 314 & 333 & 338 \\
\hline$E(\mathrm{TO} 6)$ & 366 & 360 & 370 & 370 \\
\hline$E(\mathrm{TO7})$ & 476 & 477 & 477 & 475 \\
\hline$E(\mathrm{TO} 8)$ & 523 & 516 & 517 & 522 \\
\hline$E($ T09) & 557 & 540 & 571 & 543 \\
\hline
\end{tabular}

Table 3

Detailed magnetic parameters.

\begin{tabular}{|c|c|c|c|c|}
\hline & BFO & BSFO & BGFO & BYFO \\
\hline$M_{\mathrm{r}}(\mathrm{emu} / \mathrm{g})$ & 0.009 & 0.023 & 0.011 & 0.019 \\
\hline$H_{\mathrm{c}}(\mathrm{Oe})$ & 579 & 866 & 331 & 611 \\
\hline \multicolumn{5}{|c|}{$\begin{array}{l}\text { In order to explore the microwave electromagnetic properties of samples, the air-line method was } \\
\text { employed to measure the complex permittivity and complex permeability within the } 2-18 \mathrm{GHz} \text {, as } \\
\text { shown in Fig. } 5(\mathrm{a}) \text { and (b). According to the figures, the real part of permittivity is improved for doped } \\
\text { components, and accompanied by a certain resonance at } 16 \sim 17 \mathrm{GHz} \text {. Among the samples, BGFO } \\
\text { shows better dielectric loss. The complex permittivity of all the samples is at a lower level due to } \\
\text { ceramics were prepared using rapid liquid state method. The leakage current caused by oxygen } \\
\text { vacancy is widely considered to be a major influential factor on the dielectric loss for BFO in the } \\
\text { microwave band [20, 21], and we suppose that other defects generated during the preparation process } \\
\text { will also have a negligible effect on the dielectric properties of ceramics. The feature of the complex } \\
\text { permeability for ceramics exhibits the inherent weak ferromagnetism of samples, which is consistent } \\
\text { with previous magnetic analysis. }\end{array}$} \\
\hline
\end{tabular}


A series of doped BFO ceramics were successfully synthesized by the rapid liquid state method. The participation of trivalent ions modulated the spatial structure of $\mathrm{BFO}$ to a certain extent, tilted the $\mathrm{FeO}_{6}$ octahedron. The space-modulated spiral structure of BFO was further suppressed due to the minor changes in periodic structure, which is originated from the tilting of oxygen octahedron by the doping of trivalent ions, improved ferromagnetism of doped samples. Moreover, doped samples exhibited enhanced permittivity accompanied natural resonance around $16 \sim 17 \mathrm{GHz}$, provided a feasible route to further regulate the microwave electromagnetic performance of bismuth ferrite.

\section{Declarations}

\section{Acknowledgements}

This work was supported by the National Natural Science Foundation of China (51502054), the Postdoctoral Science Foundation of China (2014M551236), the Postdoctoral Science Foundation of Heilongjiang Province (LBH-Z14083).

\section{References}

[1] Chen Z, Luo Z, Huang C, et al. Low-symmetry monoclinic phases and polarization rotation path mediated by epitaxial strain in multiferroic BiFeO3 thin films. Adv Func Mater 2011, 21: 133-138.

[2] Wu J, Fan Z, Xiao D, et al. Multiferroic bismuth ferrite-based materials for multifunctional applications: ceramic bulks, thin films and nanostructures. Prog Mater Sci 2016, 84: 335-402.

[3] Pan H, Li F, Liu Y, et al. Ultrahigh-energy density lead-free dielectric films via polymorphic nanodomain design. Science 2019, 365: 578-582.

[4] Hong $\mathrm{Y}, \mathrm{Li} \mathrm{J}, \mathrm{Bai} \mathrm{H}$, et al. Role of finite-size effect in $\mathrm{BiFeO}_{3}$ nanoparticles to enhance ferromagnetism and microwave absorption. Appl Phys Lett 2020, 116: 013103.

[5] Shirolkar MM, Dong X, Li J, et al. Observation of nanotwinning and room temperature ferromagnetism in sub- $5 \mathrm{~nm} \mathrm{BiFeO}{ }_{3}$ nanoparticles: a combined experimental and theoretical study. Phys Chem Chem Phys 2016, 18: 25409-25420.

[6] Kubel F, Schmid H. Structure of a ferroelectric and ferroelastic monodomain crystal of the perovskite $\mathrm{BiFeO}_{3}$. Acta Crystallogr B 1990, 46: 698-702.

[7] Wang J, Neaton $\mathrm{J}$, Zheng $\mathrm{H}$, et al. Epitaxial $\mathrm{BiFeO}_{3}$ multiferroic thin film heterostructures. Science 2003, 299: 1719-1722.

[8] Sosnowska I, Azuma M, Przeniosło R, et al. Crystal and magnetic structure in co-substituted $\mathrm{BiFeO}_{3}$. Inorg Chem 2013, 52: 13269-13277. 
[9] Delaire O, Stone MB, Ma J, et al. Anharmonic phonons and magnons in $\mathrm{BiFeO}_{3}$. Physl Rev B 2012, 85: 064405.

[10] Sosnowska I, Loewenhaupt M, David W, et al. Searching for the Magnetic Spiral Arrangement in $\mathrm{Bi}_{0.7} \mathrm{La}_{0.3} \mathrm{FeO}_{3}$, Materials Science Forum 1993, 133: 683-686.

[11] Tu CS, Chen PY, Chen CS, et al. Tailoring microstructure and photovoltaic effect in multiferroic Ndsubstituted $\mathrm{BiFeO}_{3}$ ceramics by processing atmosphere modification. J Eur Ceram Soc 2018, 38: 13891398.

[12] Pattanayak S, Choudhary R, Das PR, et al. Effect of Dy-substitution on structural, electrical and magnetic properties of multiferroic $\mathrm{BiFeO}_{3}$ ceramics. Ceram Int 2014, 40: 7983-7991.

[13] Wang N, Li Y, Wang F, et al. Structure, magnetic and ferroelectric properties of Sm and Sc doped $\mathrm{BiFeO}_{3}$ polycrystalline ceramics. J Alloy Compd 2019, 789: 894-903.

[14] Jena AK, Satapathy S, Mohanty J. Magnetic properties and oxygen migration induced resistive switching effect in Y substituted multiferroic bismuth ferrite. Phys Chem Chem Phys 2019, 21: 1585415860.

[15] Yang $\mathrm{CH}$, Kan D, Takeuchi I, et al. Doping $\mathrm{BiFeO}_{3}$ : approaches and enhanced functionality. Physical Chemistry Chemical Physics 2012, 14: 15953-15962.

[16] Catalan G, Scott JF. Physics and applications of bismuth ferrite. Adv Mater 2009, 21: 2463-2485.

[17] Lennox RC, Price MC, Jamieson W, et al. Strain driven structural phase transformations in dysprosium doped $\mathrm{BiFeO}_{3}$ ceramics. J Mater Chem C 2014, 2: 3345-3360.

[18] Dutta DP, Mandal B, Mukadam M, et al. Improved magnetic and ferroelectric properties of Sc and $\mathrm{Ti}$ codoped multiferroic nano $\mathrm{BiFeO}_{3}$ prepared via sonochemical synthesis. Dalton T 2014, 43: 78387846.

[19] Sharma P, Satapathy S, Varshney D, et al. Effect of sintering temperature on structure and multiferroic properties of $\mathrm{Bi}_{0.825} \mathrm{Sm}_{0.175} \mathrm{FeO}_{3}$ ceramics. Mater Chem Phys 2015, 162: 469-476.

[20] Liu X, Wang L-S, Ma Y, et al. Enhanced microwave absorption properties by tuning cation deficiency of perovskite oxides of two-dimensional $\mathrm{LaFeO}_{3} / \mathrm{C}$ composite in X-band. ACS Appl Mater Inter 2017, 9: 7601-7610.

[21] Yuan J, Hou ZL, Yang HJ, et al. High dielectric loss and microwave absorption behavior of multiferroic $\mathrm{BiFeO}_{3}$ ceramic. Ceram Int 2013, 39: 7241-7246. 


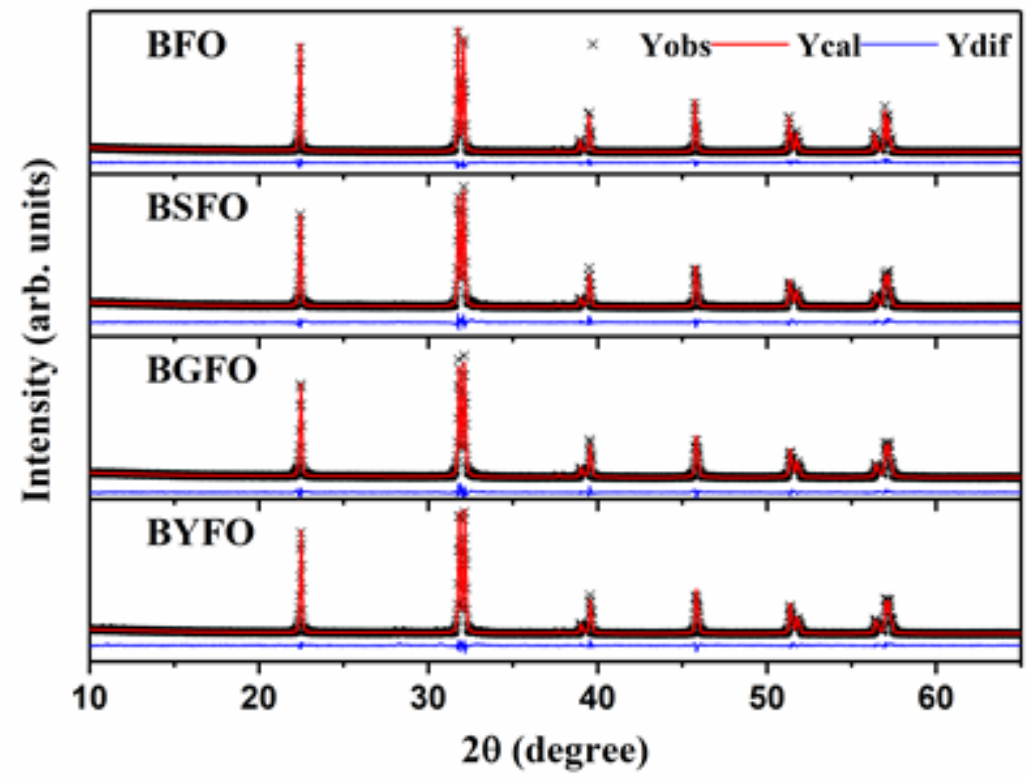

Figure 1

Rietveld-refined XRD patterns of BFO, BSFO, BGFO, and BYFO samples.
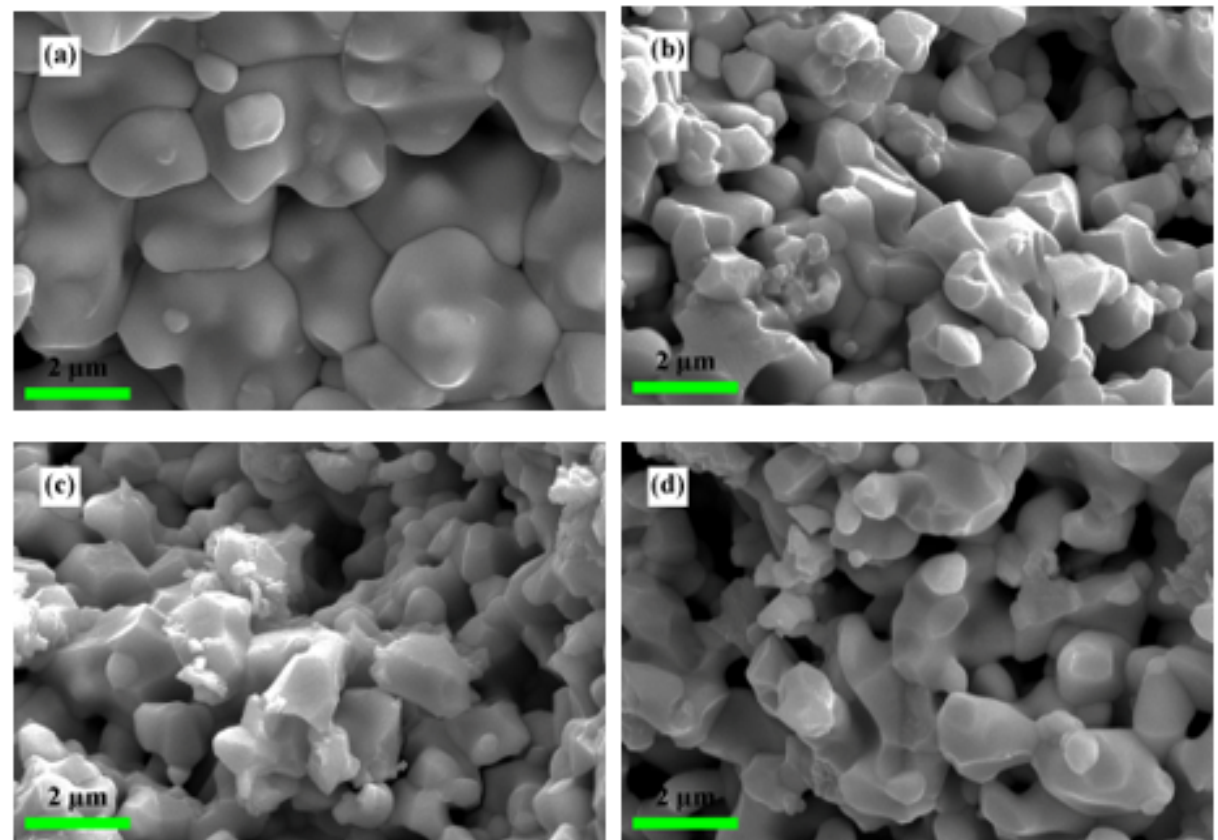

Figure 2

SEM micrographs of as-prepared ceramics: (a) BFO, (b) BSFO, (c) BGFO and (d) BYFO. 


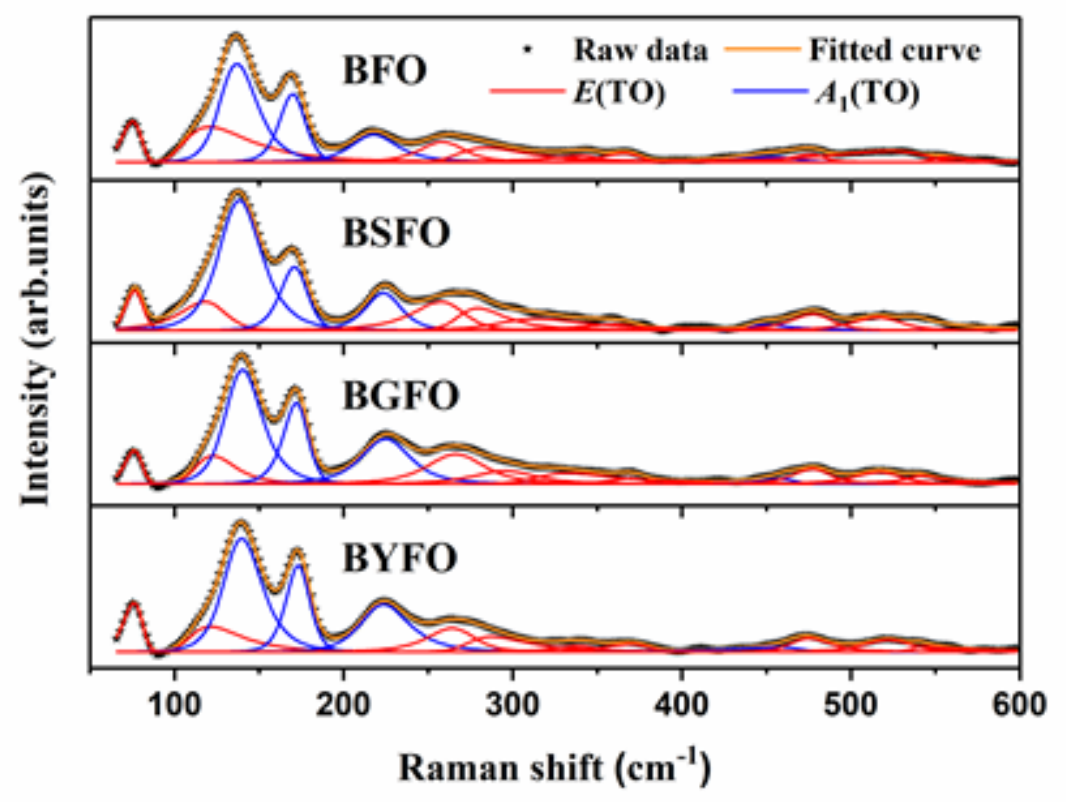

Figure 3

Deconvoluted Raman spectra of all samples.

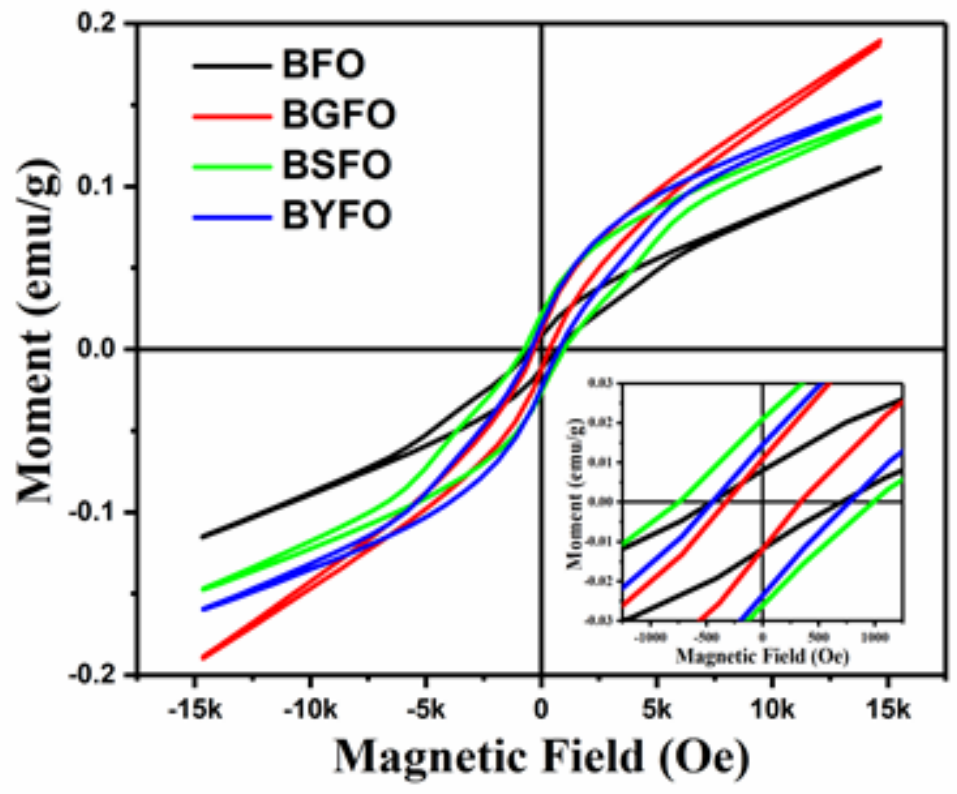

Figure 4

$\mathrm{M}-\mathrm{H}$ hysteresis loops for pure and doped BFO ceramics, and the inset depicts partial enlarged curves. 

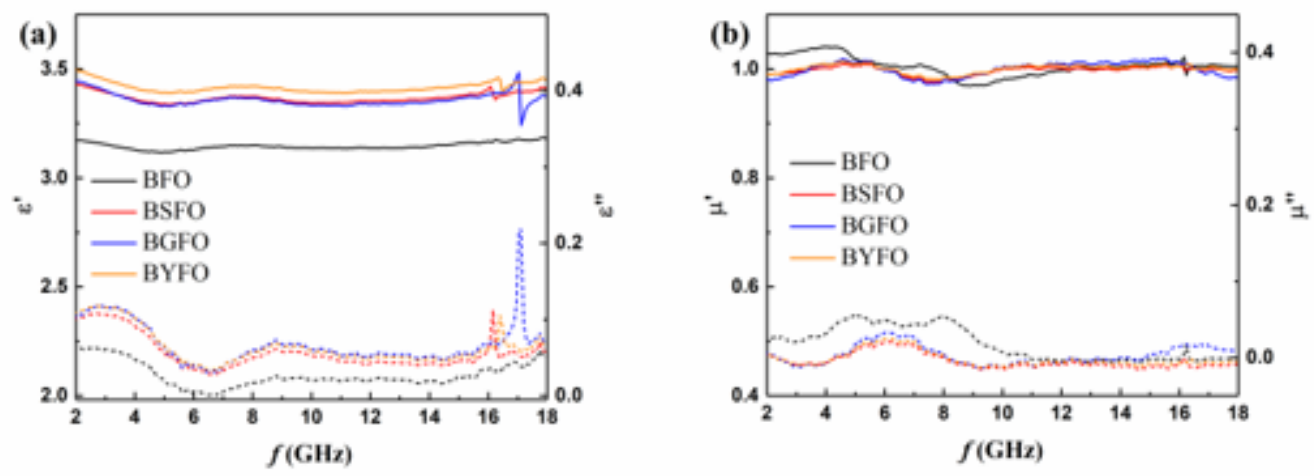

Figure 5

(a) Real and imaginary parts of permittivity and (b) real and imaginary parts of permeability of BFO, BSFO, BGFO, and BYFO, respectively.

\section{Supplementary Files}

This is a list of supplementary files associated with this preprint. Click to download.

- Equation.pdf 